\title{
SCIENCE
}

FridaY, DeCEMBer 27, 1912
CONTENTS
The Artificial Ripening of Bitter Fruits:

Professor Francis E. Lloyd .......... 879

University Registration Statistics: ProfesSOR RUdoLF Tombo, JR. ........... 887

The Fur Seal Census: George ARchibald Clark ....................... 894

The Fur Seal Mortality of the Pribilof Rookeries in the Absence of Pelagic Sealing: M. C. Marsh $\ldots \ldots \ldots \ldots \ldots \ldots \ldots \ldots . \ldots . \ldots 97$

Memorial of a Centenary ............ 898

Scientific Notes and News ............ 900

University and Educational News ....... 902

Discussion and Correspondence:-

Philippine Sharks: DR. H. M. SмIтH. Berardius Bairdii in Japan: Dr. Roy C. ANDREWS. On Citing the Types of New Genera: Professor T. D. A. Cockerell. In the Interests of Better Speaking: X. Y. Z. .................... 902

Scientific Books:-

Moore's Principles of Microbiology: ProFEsSOR F. P. Gorham. Conn's Biology: M. M. Franz's Handbook of Mental Examination Methods: F. L. W. Ries on Building Stones and Clay Products: Dr. Geo. P. MERRILL ................ 904

Special Articles:-

The Relationships of the Chestnut Blight

Fungus: DR. G. P. ClintoN ......... 907

The Convocation Week Meeting of Scientifio Societies ....................... 914

MSS. intended for publication and books, etc., intended for review should be sent to Professor J. McKeen Cattell, Garrisonon-Hudson, N. Y.
THE ARTIFICIAL RIPENING OF BITTER FRUITS ${ }^{1}$

THIs subject has been chosen not only because of a certain intrinsic interest which I trust will presently be admitted, but because also it serves to illustrate the important contention that the problems of pure and applied science go back for their solution to the same fundamental principles. It is true that empiricism has solved, in a measure, many practical problems, and that, indeed, science has grown out of empiricism. But science in her turn leads more rapidly and surely to the goal which is sought, for the simple reason that she explains why things happen as they do. For a single and almost overworked example, the ancients knew that peas and clovers enriched the soil, and this knowledge led to the practise of rotation in the planting of crops. But it is very recent knowledge that this behavior is due to the peculiar relations of certain bacteria to a limited group of the higher plants known collectively as the legumes, by which the free nitrogen of the air is made available to the latter. The economic salvation of immense areas, yet to be accomplished, may now be compassed with this knowledge-a very practical outcome. On the other hand, the understanding of the nitrogen relations of plants has stimulated the chemist to discover a method, and if possible an economical method, of fixing atmospheric nitrogen, so that this vast storehouse of material may be rendered more available, the solution of which problem could not by any chance have been attained except by.

1 The university lecture, October 8, 1912. 
the application of highly theoretical knowledge.

A further, but less widely appreciated, example of the same thing is afforded by the present subject, namely, the control of the certain ripening processes in bitter, or rather astringent fruits. We shall see, as the details are unfolded, that here too empiricism has preceded science, but that science is at the moment endeavoring to explain experience, with the result that, while problems of very great practical importance are being solved, new facts of still greater theoretical weight are being discovered.

Astringency in fruits and other plant parts is due to the presence of tannin in some form, very frequently tannic acid. It will suffice here to speak of it simply as tannin. Certain kinds of bark and other parts of many plants contain this substance in such quantities that they are used for the tanning of hides and for the extraction of tannin for other purposes, some of which are medicinal. The usefulness of tannin in the arts depends on its peculiar property of being able to enter into combination with many other substances, such as proteins, gelatins, mucilages and the like, to form complexes (or compounds in a loose sense) which have in common the peculiar property of resisting agents of decay. This fact is perfectly well known and tannin has been made use of since time immemorial as a preservative of material exposed to conditions favorable to decay, such as sails, fishing tackle and the like.

But if one wishes to have a more vivid impression of this peculiarity of tannin seen in its ready combination with colloidal materials, one needs but to take into the mouth a few drops of a solution of tannin, or to bite into an astringent fruit, such as the persimmon, or an unripe banana. The tannin in these will quickly attack and combine with the skin of the mouth, and produce the drawing or puckering effect familiar to all. The same experience is had on drinking tea which has been too long standing. What happens in the mouth may be accurately and simply described as a rapid manufacture of a thin coating of leather over the mucous membranes.

Another peculiarity of these tannin complexes, such as leather and the like which it is necessary to mention briefly, is this, namely, that one may easily extract with water a certain amount of the tannin without any obvious change in the physical character of the complex. If we soak leather in water, we can show that some of the tannin has come out. We may do this repeatedly, and always get some but again always, a decreasing amount of tannin. An analogous example is afforded by certain dyes which stain the cotton fiber but which may be extracted in repeatedly diminishing quantities by water. Such complexes are said to arise by adsorption; they are not, at any rate, chemical compounds in the ordinary sense. In this connection our attention should be drawn to another fact of prime importance in what follows. It is that dyes are held more tenaciously by coagulable substances, such as the white of egg, when they are in a state of coagulation than when not, so that, given a certain amount of dye and a certain amount of the albumin (or whatever other substance may be used), a less amount of the dye can be extracted by water if the albumin has been coagulated by heat-as happens when an egg is cooked-than otherwise. I have shown that tannin in its relation with a coagulable substance in the persimmon, analogous to the white of egg, acts in the same way. The significance of this in relation to non-astringency of fruits will be indicated as we proceed.

The term colloid has already been used. 
Inasmuch as the living body, whether of the plant or of the animal, is made up, aside from the water content, very largely of colloids, I must venture, at the risk of appear. ing to dwell overmuch upon very elementary matters, to draw attention to certain of their characteristics. In negative fashion, a colloid may be described as a substance which does not crystallize, and this feature serves to contrast it with other sub. stances, such as salts, sugars, etc., which, upon going out of solution, assume geometrical forms. It is more difficult to define colloid in positive terms, but fortunately we are all of us familiar enough with them so that we do not need a formal description. Glue, gelatin, mucilage are examples. Tannin, which claims our especial notice at this time, is also a colloid. When colloids are dissolved in water, they break up into particles which are far too small to be seen with the naked eye, but which are very much larger than the particles in a solution of a crystalloid. These may be identical with the molecules, or still smaller, when they represent the ions, or grosser components of the molecule small beyond even the strongest powers of the microscope. Colloids, however, in many cases, may in their dissolved condition be seen by means of the ultramicroscope, when they appear as minute brilliantly illuminated particles (suspensoids). One may understand this by recalling that a very small mirror at a great distance can be seen when it is caused to reflect the sunlight into the eye of the observer. The ultramicroscope therefore enables us in many instances to see what goes on in solutions of colloids. For example, it makes it possible to watch the process of coagulation in those colloids in which coagulation is possible. Thus, if we examine a wèk casein solution-we can make such by thinning skimmed milk with water-we see a very pandemonium of dancing illumined particles. These remain in constant motion, flying hither and yon at a rate of speed too great to follow with the eye. If now we add a minute amount of an acid, the particles may be observed to hit one another and to remain in contact, so forming a continuous mass or apparently continuous, since we know that water is held within the coagulum. Quite similar appearances may be had by adding a solution of tannin to one of gelatin.

If, however, before adding acid to the casein solution, we add a little mucilage, the coagulation may be prevented. This illustrates the principle of colloidal protection-in this instance the mucilage protects the casein from the action of the acid. I have shown that a similar relation exists between tannin and mucilage as against alkaloids. Tannin immediately precipitates an alkaloid, such as antipyrin, in solution in water. When, however, a mucilage has previously been added, the precipitation is prevented. If, therefore, on adding an alkaloid to a solution of tannin we get no precipitate, we must argue that there is a third substance present which protects the tannin. Such a condition will be shown to occur in the fruits with which we concern ourselves to-day.

We now turn to examine typical examples of fruits which, before entering the condition regarded as edible, are highly astringent, but which, when "ripe," appear to be entirely devoid of the astringent principle, tannin. I use this wording advisedly, since the fact is that such fruits contain quite as much tannin when nonastringent as before. What has become of the tannin I propose to show you. The examples in question are the date of Arabia and Africa, the staple food product of the Arab, and the persimmons of eastern Asia and of North America. 
The fruit of the date palm is hard, green and highly astringent, the approximately oval shape and the size varying in the different varieties, of which there are many. When ripe they may be dry or very soft and syrupy, again according to the variety. The soft dates when ripe can not be transported unless they are dried and packed, and it is in this condition that we are familiar with this fruit. In order to market the soft varieties so that they may be consumed in the fresh condition, the Arabs have practised for many centuries the art of artificial ripening. This consists in exposing the bunches of unripe fruit to the vapors of vinegar, of which the active agent appears to be the acetic acid, judging from the experiments of Vinson, of the Arizona Agricultural Experiment Station. Among other changes which take place during the artificially induced ripening period, the most obvious is the rapid disappearance of astringency, so that the quite inedible fruit of to-day is ready to the palate on the morrow or at latest the following day. Vinson further found that there is a great variety of chemicals which can produce the same result; so various in kind indeed are the chemicals that the changes in the fruit can not be explained as ordinary chemical reactions. Supranormal temperatures up to 70 degrees Fahr. may also be used, but higher temperatures kill the fruit, after which it is impossible to render them non-astringent. There is, however, a distinction to be drawn at this point between the effects of certain ripening agents and others. Thus, when some chemical substances, such as acetic acid, nitrous ether, etc., are used, the tannin changes from being colorless to red, indicating that it has been oxidized. Oxidized tannin is tasteless, so that in such case the non-astringency is readily explained. If heat or alcohol be used, the tannin remains colorless, and as such is astringent, so that, if it can not be detected by the tongue, this is the result of some other condition than oxidation. Indeed the evidence is well nigh conclusive that during the ripening of the date the tannin remains unchanged chemically, but that its collophysical relations have been changed. That it is still present may at once be demonstrated by the use of suitable chemical reagents - their suitability depending on whether the reaction produces a color change or not. Thus the salts of iron and other metals produce the corresponding color changes; alkaloids produce no change. It has been pointed out before, however, that the reaction of alkaloids and tannin may be prevented by means of a protector and this leads us to the conclusion that non-astringency in the ripe date is due not to the destruction of the tannin or to any chemical change in it, but to the presence of an efficient protector. Further evidence for this will be given further on.

The persimmon occurs wild as a single recognized species in North America, and the fruit is known in the south as the " 'possum persimmon" for the reason that the opossum fattens on it in the autumn. In China and Japan there are numerous cultural varieties, most of which resemble a ripe tomato in size, shape and color. Certain kinds lose their astringency before they soften, and can be eaten as we eat apples, biting into a firm flesh. Others become edible only after becoming quite soft and watery, so that a spoon becomes an appropriate implement for managing them. Still another kind, described as an "astringent variety" is used as the source of a fluid, called "kaki-no-shibu," obtained by mashing up the fruits in water and allowing the mash to ferment. The product is used for waterproofing fishing lines and nets, and for coating wrapping paper, 
paper umbrellas and similar services ${ }^{2}-$ examples of the preserving effect of tannin. The peculiar properties of kaki-no-shibu, however, as the Japanese chemist Ishikawa suspected years ago, is not due to the tannin content alone, but to the joint action, as I believe, of the tannin and another substance of mucilage-like character with which the tannin is combined much as it is with hide to make leather.

We are concerned here, however, with those edible sorts which do not become nonastringent without at the same time softening. These when quite ripe can only by courtesy be called edible-decayed would better describe their condition. They have lost a fine aroma and a delicate sweetness which, coupled with a crisp firmness, would, in the absence of tannin, make up the qualities of a delicious fruit, as those know who have eaten them after being ripened artificially either in Japan or at home. This is accomplished by the Japanese, as it has been for centuries doubtless, by packing the hard, unripe fruit in freshly emptied tubs, in which sakè, the national whisky of that people, has been kept. A fully packed tub is tightly closed and the contained air in them displaced ${ }^{3}$ by blowing through a small hole, the air escaping by another. The openings are then plugged so that the package is made airtight. In the course of a few days, the length of time depending upon the variety under process, the fruit becomes edible without losing its firmness. This hastening of one of several independent processes, spoken of collectively as ripening, result-

\footnotetext{
${ }^{2}$ Bishop Heber Hamilton informs me that kakino-shibu is used also for painting exposed woodwork, but is efficient only for a year. When applied it is colorless, but turns red in a few daysevidently by oxidation of the tannin.

${ }^{3}$ According to some accounts, this is done when reclosing the package after it has been opened to test the fruit.
}

ing in non-astringency, has been regarded as due to the action of some constituent of the sakè, with which the wood of the cask is of course soaked. This may be either the alcohol or a slight amount of acetic acid. I have argued, from experimental data, that it is the alcohol which, by stimulating the fruit to respiration in excess of the normal, quickly causes a formation of carbonic acid gas in addition to that which is introduced by the breath. At any rate, there is little doubt that the carbonic acid gas is the active agent, since Gore, of the Bureau of Chemistry, U. S. Department of Agriculture, found that certain varieties of persimmons grown in the United States could be treated with carbon dioxide at normal pressure with results quite similar to those obtained by the Japanese with their ancient but empirical method. Gore's experiments were in part repeated by myself at the State Experiment Station of Alabama, with like outcome. It was found that the variety known as Hyakume $e^{4}$ grown on the station grounds if exposed for about eight days to an atmosphere of pure carbon dioxide, loses its bitterness while still remaining firm and crisp, and retaining its aroma and sweetness. The fruit so treated was most excellent to the taste, as testified to by a number of persons whose skepticism regarding the edibility of a hard persimmon had been begotten of much experience, and whose testimony was therefore of the greater value.

At this point a question arose. If a pure atmosphere of carbon dioxide at ordinary pressure induces the already described result, why should not an increased dosage effect the same result more rapidly? To attain to the answer I exposed the same variety of fruit to twice the amount of the

- There are two varieties of Hyakume recognized, one of which is astringent until softening intervenes, while the other loses its astringency while still firm. 
gas by using a metal tank which would maintain the required pressure. Into this was forced the gas to an indicated pressure of 15 pounds, which means that two volumes of the gas were compressed into the enclosed space of the apparatus. Under these conditions the fruit became quite edible in less than 46 hours. That is, the time required to render the fruit non-astringent under normal atmospheric pressure was reduced to one fourth that time, or rather less, as it later appeared, by increasing the pressure twice. This was in the autumn of 1911, at the time when no further experiments were possible. During the early part of September, 1912, the experimentation with supranormal pressures was renewed. A special apparatus had already been devised, composed of a piece of four-inch gas pipe, suitably capped, and supplied with a pressure gauge and with outlets guarded by gas cocks. This device enabled me to try the effects of pressure reaching up to 90 pounds, or seven times the original dosage. It is interesting to note that this high pressure kills the fruit in a few hours, so that it becomes watery and unattractive. It is nevertheless non-astringent. The effect, however, of 45 and of 15 pounds pressure separately were determined with considerable accuracy, with the following result. The fruit exposed to 15 pounds pressure became non-astringent in about 36 hours; that subjected to 45 pounds in about 15 hours. We may say, therefore, that, as the pressure of the carbon dioxid increases, the period of time required for causing the apparent disappearance of the tannin is decreased, and that the pressure goes up much less rapidly than the period is decreased. To make assurance doubly sure, the fruits which were used in the experiment which I have just briefly summarized ranged in degree of maturity from being entirely green, of the green of grass, to orange-yellow, the next to final color stage of ripening, and it eventuated that fruits which are entirely green can be rendered non-astringent in scarcely less time than those much nearer maturity, thus leaving no doubt of the significance of the experiments. To state these results in everyday terms of practical economics, we can say that it is now possible to ship, say on September first, green or near-green persimmons of the variety mentioned from Alabama to Montreal, where they should arrive in good condition, hard and without bruise or other blemish, on September third. Being, however, quite inedible, and with a prospect of remaining so for a month, or even longer, if kept in cold storage, the fruit is placed in a very simple and cheap apparatus, and subjected to 45 pounds pressure of carbon dioxid. The gas of course costs very little, and can be easily obtained-it is used in every soda water fountain. The next morning, that of September fourth, the fruits may be marketed, and if the fruit dealers know human nature as well as they appear to, the fruit would be readily disposed of at a high price, if properly displayed and advertised. Recurring to the fruit of the date palm, the methods which have been elaborated in Arizona, when finally given finesse, will make it possible to utilize vast desert areas for the culture of the date palm, and so making an otherwise useless waste contribute to human welfare. Of the cultivated persimmon something the same may be said. In California the culture of this tree has fallen into desuetude, and in the southeast hundreds of acres of persimmons are practically of no profit, for the reason that a method of marketing edible fruit has been wanting until now. It remains only to perfect in detail for the several varieties of 
the fruit, the methods used by Gore ${ }^{5}$ and as here outlined, when this desideratum will be compassed.

Having justified the scientific method from the practical point of view, as I believe to have been done, may I finally ask your attention to the further question which you will be sure to ask, namely, why carbon dioxide should act as it does. Why should a fruit which would remain under normal conditions inedible for its bitterness during a month or more, become quite edible in a week with a single dose of a certain gas, in 36 hours if the dose is doubled and in 15 hours if quadrupled? Let me prepare your minds for the answer by reminding you of the very familiar fact that it takes a good deal longer to cook an egg to hardness if a temperature considerably below boiling point is used than if it is subjected to 212 degrees or over. With this in mind, our attention may be directed to a few points eoncerning the structure of the fruits of which we have been speaking.

The edible flesh or pulp is composed of a great number of minute cellulose sacs (cells) each containing its quantum of living material as an inner lining, and this in turn filled with sap which is water with varying amounts of substances, such as mucilage, sugars, and salts, in solution. In both the persimmon and in the date, these sacs are of two quite distinct kinds, those from which tannin is absent, and which are relatively small; and those in which tannin occurs admixed with other substances in the sap. The tannin sacs are quite large, and may be readily distinguished by the unaided eye. You may see them in the ordinary dried dates of commerce as a layer of clear brown particles just beneath the somewhat tough skin. The color, however, is due to the oxidation of the tannin-in the fresh condition they

\footnotetext{
${ }^{5}$ Bulls. 141, 155, Bur. Chem., U. S. Dept. Agri.
}

are colorless, and can be recognized only by special means, that is, by applying suitable reagents which cause color changes in the tannin.

If now we choose a persimmon which softens before it loses its astringency, it is possible to isolate from the pulp single tannin sacs, which may then be examined under the microscope. If uninjured-if the cellulose membrane is not rupturedthe watery contents will glisten with a satiny sheen. On adding water so that the tannin sac is surrounded by it, the sac absorbs water and bursts, and the contents ooze out. This simple fact of bursting in consequence of the absorption of water proves conclusively that there is something more than tannin present, as tannin in solution can not absorb water sufficiently to produce such an effect. Sugar or salts might, if in sufficient quantity; but we can prove in another way that the substance in question is of neither of these classes of material, for it is capable of coagulation, in much the fashion that, as every housewife knows, we may coagulate jelly by cooling it, or an egg by boiling it. In the case of our tannin sac material, we may use heat, or a variety of chemical substances. Of these I shall, for the present purpose, mention but one class, namely, the alkaloids, such as antipyrine, quinine, etc. If a solution of any of these be applied in the room of water, the jelly-like mass may swell somewhat at first, but soon becomes hard and rigid, giving off water and shrinking accordingly. At the same time, however, within the interior of the coagulated mass there appears a coarse white granulation, which is caused by the union of the alkaloid with tannin within the jelly. This relation of tannin to the jelly is brought out still more strikingly if we examine, in the same way, a tannin sac which has been taken from a fruit which has become nearly 
non-astringent. If we take a portion of the pulp into the mouth we do not at first notice the puckery effect. In a few moments, however, this develops and becomes more and more pronounced during several minutes. Under the microscope we can actually watch the process. On adding water to some suitably isolated tannin sacs, the contents swell more slowly than before, but ultimately burst out and form a bubble-like mass on the side of the sac. In a few moments a granular veil is seen developing just beyond the surface of the protrusion, gradually increasing in size and moving away further and further. It would take us too far afield to explain why this peculiar behavior, so it must suffice to say that it is due to escaping tannin, which leaves the mass out of which it has escaped unaffected in shape and size. The matter is quite analogous to the washing out of color from cloth: the color goes, the cloth remains. Repeating our experiment with antipyrine, we now find that, while coagulation takes place, the amount of shrinkage is less than before, and the action of the reagent on the tannin within the mass is less apparent for the reason that the granulations are smaller.

If finally we treat a tannin sac from a quite non-astringent fruit in the same way we shall find that it will swell but little or none at all in water, that the alkaloid causes little shrinkage if any, and that the tannin reaction does not take place at all. That the tannin is still there is, however, apparent if we use other reagents, all of which nevertheless act much more slowly than they are known to do when unripe material is tested by them. The conclusion therefore to which $I$ arrive is that the reason we do not taste the tannin in the completely ripened fruit is not because it is not there, but because the jelly-like material which occurs in the tannin sacs along with the tannin itself becomes coagulated during the ripening process, so that the tannin may not escape from it except at a very slow rate-too slowly far to be detected even by the delicate membranes of the mouth. To recall what was said earlier in the hour, the tannin is protected by the jelly, so that the alkaloid can not act on it -and this the more efficiently as the coagulation is the more complete. Tannin itself, on the other hand, is not a coagulable material. Although a colloid, it does not have the physical properties of a jelly or mucilage. In a word, we have in the tannin masses of the ripe fruit a sort of vegetable leather, which, like ordinary leather, gives up its tannin only very slowly, as shown by long exposure to water. I have tannin sacs of the persimmon which have been lying in water for over two years, but, aside from the loss of tannin, they remain quite unchanged, and will doubtless do so for years to come.

But what of the relation of all this to the carbon dioxid? We can form some notion of the matter if we step aside to enquire somewhat into the behavior of this gas. The more ordinary name, carbonic acid gas, indicates that it is an acid, and it is therefore of a class of substances which may exert a coagulating (or flocculating) influence upon various colloids. For a single example, carbon dioxide has been found recently to cause the coagulation of the milk or latex of india-rubber trees. In coagulated latex, the india-rubber occurs as minute droplets which remain individual and separate until some coagulating agent has its way, when they run together to form a continuous mass of india-rubber. This is only one example of the effect of carbon dioxid upon substances in the colloidal state, and by it we are led to suspect that its rôle in the artificial ripening of dates and persimmons is referable to its 
coagulating power, a suspicion which is strengthened by the fact that the more there is available, as when it is supplied under pressure, the quicker is the effect. Some may look askance at so simple an explanation when so complicated a phenomenon is involved, and they are quite justified in doing so. This explanation is advanced not as final, but as a theory well worthy further examination by the experimental method, the method by which only can science be advanced, my purpose here being to discover a problem in science as it confronts the investigator rather than to lead you on the smooth, well-worn but less picturesque and romantic road in the domain of the already known. Let me, therefore, not tax your patience too far, enough, however, to allow me to reaffirm that the problems of science are not of mere academic interest; and that sooner or later they relate themselves to human life. The problem which has been outlined illustrates this principle, and it is one which I venture to assert is well worth the severe application of the investigator, entrenching as it does on that field of the physiology of the obscure processes of respiration, digestion, enzymatic action, the relations of crystalloids and colloids and the like-in short on that field where the physiology of living things, whether of animals or plants, overlaps the as yet undeveloped knowledge of collochemistry, a field surrounded by a wide horizon of the unknown, to pass which even with a stumbling tread requires a sure faith in the strength of the staff of scientific method.

Francis E. Lloyd

\section{MCGILL UNIVERSITY}

UNIVERSITY REGISTRATION STATISTICS

THE registration returns for November 1 , 1912, of twenty-nine of the leading universities of the country will be found tabu- lated on the following page. Specific attention should be called to the fact that these universities are neither the twentynine largest universities of the country in point of attendance nor necessarily the twenty-nine leading universities, nor is there any desire on the part of the compiler to insist upon a quantitative standard as the only proper basis for comparison of our institutions of higher learning. Five institutions exhibit a decrease in the total enrollment (including the summer session), namely, Cornell, Illinois, Iowa, Johns Hopkins and Pennsylvania, while four institutions showed a loss in the total enrollment last year, and three in 1910 and four in 1909. The largest gains in terms of student units, including the summer attendance, but making due allowance by deduction for the summer session students who returned for instruction in the fall, were registered by Columbia (1,069), California (733), Minnesota (515), New York University (488), Texas (475), Nebraska (391) and Harvard (303). Last year there were four institutions that showed a gain of over three hundred students, namely, California, Columbia, Cornell and Ohio State, whereas in 1911 and in 1910 there were seven institutions that registered such an increase. Omitting the summer session attendance, the largest gains have been made by Indiana (990), Chicago (700), California (690), Columbia (484), New York University (375), Nebraska (337), Texas (318), Cornell (284), Northwestern (232) and Syracuse (209). It will thus be seen that this year ten institutions exhibited an inerease of over two hundred students in the fall attendance, as against four in 1911, seven in 1910 and eleven in 1909. It will be observed that of these institutions four are in the east, five in the west and one is in the south.

According to the figures for 1912, the 